\title{
PSICODIAGNÓSTICO NA UNIDADE DE INTERNAÇÃO PSIQUIÁTRICA DE UM HOSPITAL PÚBLICO
}

\section{PSYCHODIAGNOSIS IN A PUBLIC PSYCHIATRIC UNIT OF A GENERAL HOSPITAL}

Clin Biomed Res. 2021;41(2):126-132

1 Residência Integrada Multiprofissional da Saúde, Hospital de Clínicas de Porto Alegre. Porto Alegre, RS, Brasil.

2 Departamento de Psicologia, Universidade Federal de Ciências da Saúde de Porto Alegre (UFCSPA). Porto Alegre, RS, Brasil.

3 Serviço de Psicologia, Hospital de Clínicas de Porto Alegre (HCPA). Porto Alegre, RS, Brasil.

Autor correspondente: Juliana Unis Castan jcastan@hcpa.edu.br Hospital de Clínicas de Porto Alegre (HCPA)

Rua Ramiro Barcelos, 2350 90035903, Porto Alegre, RS, Brasil.

\section{Larissa O'nill de Avila Pereira', Joana Correa de Magalhães Narvaez², Juliana Unis Castan ${ }^{3}$}

\section{RESUMO}

Introdução: Este estudo caracterizou pacientes adultos que tiveram solicitação de psicodiagnóstico enquanto estavam internados em leitos do Sistema Único de Saúde (SUS) em uma unidade psiquiátrica de um hospital geral universitário de Porto Alegre no ano de 2017. Com o intuito de subsidiar o planejamento deste serviço, assim como a discussão com as equipes assistenciais a respeito das indicações para a solicitação, buscou-se conhecer as características tanto dos pacientes beneficiados por este exame como de suas internações. Traçou-se o perfil demográfico, identificou-se as especificidades das internações quanto ao motivo, tempo de permanência e diagnóstico psiquiátrico, e observou-se as características da própria avaliação psicológica.

Método: Estudo exploratório, quantitativo e transversal, em que os dados foram resgatados dos prontuários de forma retrospectiva. A análise dos dados foi feita de forma descritiva e inferencial, com auxilio do SPSS versão 16.0.

Resultados: Dos 247 pacientes internados, 17,8\% tiveram solicitação de psicodiagnóstico, sendo que 93,2\% (41) tiveram o processo concluído. A demanda principal foi para pacientes adultos jovens, possivelmente em primeira internação psiquiátrica e em processo de avaliação diagnóstica, um dos possíveis objetivos de uma internação psiquiátrica.

Conclusão: O psicodiagnóstico auxilia no entendimento dinâmico do paciente; entretanto, este é um recurso técnico que precisa ser usado de forma estratégica durante a internação psiquiátrica por conta dos fatores que podem envolver desde o funcionamento da própria unidade até o estado mental e emocional do indivíduo em momento de crise.

Palavras-chave: Técnicas psicológicas; Unidade hospitalar de psiquiatria; Saúde mental; Sistema único de saúde.

\section{ABSTRACT}

Introduction: This study characterized adult patients that had indication for psychodiagnosis while in a public psychiatric unit in a general university hospital in Porto Alegre, southern Brazil, in 2017. With the purposes of encouraging the discussion about psychodiagnosis indication among care teams and planning for this service, this study sought a better comprehension of patients' characteristics and their hospitalizations. Demographics, profile of the period of hospitalization, including reason for admission, length of stay, and psychiatric diagnosis, and characteristics of psychological assessment were described.

Methods: This exploratory, quantitative, and cross-sectional study retrieved data from medical records retrospectively. Data were then analyzed descriptively and inferentially with SPSS, version 16.0.

Results: Of 247 hospitalized patients, $17.8 \%$ had indication for psychodiagnosis, and $93.2 \%(41)$ had the procedure completed. The group that demanded most was 
young adults, possibly in their first psychiatric hospitalization and in the process of psychiatric assessment, one of the main objectives of a psychiatric admission.

Conclusion: Psychodiagnosis helps to understand the dynamics of a patient; however, it should be used with caution in psychiatric hospitalization because of factors ranging from the operation of the unit itself to the mental and emotional state of a patient during an acute crisis.

Keywords: Psychological techniques; Psychiatric department, hospital; Mental health; Brazilian unified health system.

\section{INTRODUÇÃO}

A Lei Antimanicomial 10.216 dispõe sobre as diretrizes de cuidado às pessoas com transtornos mentais e redireciona o modelo assistencial em saúde mental. Esta lei, além de preconizar tratamento igualitário, estimula a extinção dos manicômios ${ }^{1}$. Busca-se a transição de um modelo baseado no asilamento para um que valorize a reabilitação psicossocial e o resgate da cidadania. Os hospitais psiquiátricos deixam de ser o centro do cuidado aos pacientes com transtornos mentais, resultando em uma rede mais complexa de serviços como Centros de Atenção Psicossocial (CAPS), ambulatórios e leitos em hospitais gerais ${ }^{2}$.

A internação psiquiátrica em hospitais gerais é indicada em situações psiquiátricas agudas graves, apresentando risco de auto ou heteroagressão, exposição moral e/ou incapacidade grave de autocuidado, associado ao esgotamento dos recursos extra-hospitalares ${ }^{3}$. Com períodos mais curtos de hospitalização e caráter protetivo, é um recurso para um momento de maior vulnerabilidade do paciente e de sua rede. Visa à estabilização dos sintomas, minimização de riscos, estabelecimento de um diagnóstico psiquiátrico e ajuste medicamentoso ${ }^{2}$, além do planejamento terapêutico, considerando recursos locais e características individuais, da família e da comunidade.

A avaliação psicológica é um recurso na definição diagnóstica, um dos objetivos de uma internação psiquiátrica. É um processo estruturado de investigação de fenômenos psicológicos, que se utiliza de técnicas e instrumentos, podendo ser considerado um exame dentro da área da Psicologia, também chamado de psicodiagnóstico. Permite acesso ao funcionamento global do paciente, integrando capacidade intelectual, aspectos emocionais e comportamentais, auxiliando, assim, na exploração de hipóteses diagnósticas e no planejamento terapêutico ${ }^{4-8}$.

Em 2012, surgiu a campanha internacional Choosing Wisely, conduzida pela Fundação norte-americana American Board of Internal Medicine. A campanha estimula o uso racional de recursos de diagnóstico e de tratamento, tendo por objetivo conscientizar as equipes médicas em relação ao custo beneficio de exames para a qualidade do cuidado em saúde ${ }^{9,10}$. Esta estratégia pretende auxiliar no estabelecimento de critérios e demandas específicas que justifiquem as solicitações de exames e procedimento; no caso da Psicologia, pode-se pensar no exame de psicodiagnóstico.

Assim, considerando a necessidade de um melhor planejamento do uso de recursos assistenciais e a escassez de estudos que caracterizem a população que se beneficia do psicodiagnóstico durante internação psiquiátrica, este estudo tem o intuito de caracterizar pacientes adultos que realizaram psicodiagnóstico durante internação psiquiátrica em leitos do Sistema Único de Saúde (SUS) em um hospital geral universitário de Porto Alegre, considerando variáveis demográficas e características tanto da internação como do psicodiagnóstico. Acredita-se que este conhecimento é o primeiro passo para melhor planejamento deste serviço: É preciso conhecer a demanda e população atendida para planejar intervenções de forma eficiente.

\section{MÉTODO}

O estudo é exploratório, transversal e quantitativo. A amostra retrospectiva constituiu-se de pacientes com 18 anos ou mais, internados em leitos SUS de um hospital geral universitário de Porto Alegre, que realizaram o psicodiagnóstico. O hospital em questão conta com 26 leitos psiquiátricos para adultos destinados ao SUS.

O período de análise foi de 01 de janeiro a 31 de dezembro de 2017. Os dados foram coletados através de solicitação ao Serviço de Tecnologia de Informação, em que se especificaram os campos a serem resgatados dos prontuários eletrônicos. As variáveis coletadas foram: dados demográficos (data de nascimento, escolaridade, sexo, estado civil); informações referentes a internações (data da internação e alta, queixa principal, diagnóstico registrado na internação e na alta); e informações referentes ao psicodiagnóstico (data da solicitação, data da liberação no sistema, cancelamentos, motivo de solicitação, número de consultas e de pessoas entrevistadas, Quoeficiente de Inteligência Verbal, de Execução e Total).

A análise dos dados foi feita de forma descritiva e inferencial, com auxilio do Statistical Package for Social Science for Windows (SPSS) versão 16.0. De acordo com a natureza das variáveis, obteve-se 
frequência e percentual, além das médias e desvio padrão. Foram utilizados os testes Exato de Fisher, Mann-Whitney e Qui-Quadrado com correção de continuidade quando necessário, com nível de significância de $5 \%$, para comparar o grupo que teve solicitação de psicodiagnóstico, em relação ao grupo de pacientes que não teve.

Este estudo integra um projeto intitulado Caracterização da demanda de avaliação psicodiagnóstica e implementação de gerenciamento e fluxos no contexto de um hospital universitário, registrado na Plataforma Brasil sob o número CAAE 71560717.3.0000.5327 e aprovado pelo Comitê de Ética em Pesquisa da instituição na qual foi desenvolvido. As pesquisadoras assinaram o Termo de Compromisso para Utilização de Dados da Instituição, comprometendo-se a preservar a privacidade dos pacientes.

\section{RESULTADOS}

No ano de 2017, 253 pacientes internaram na Unidade de Psiquiatria. Seis pacientes permaneceram menos de 48 horas, tendo alta por solicitação do familiar responsável. Dos 247 pacientes, 17,8\% (44 pacientes) tiveram solicitação de psicodiagnóstico. Destes, $93,2 \%$ (41) tiveram o processo concluído com o laudo liberado no sistema e $6,8 \%$ (3) foram cancelados, devido a realização do exame em outra instituição, alta do paciente ou cancelamento pela equipe médica.

\section{Dados demográficos}

Idade, sexo, estado civil e escolaridade da amostra podem ser conferidos na Tabela 1. Comparando as características demográficas do grupo de quem realizou psicodiagnóstico e do grupo de quem não realizou este exame, houve diferença significativa apenas com relação à idade, conforme resultados do teste Mann Whitney $(p<0.001)$. Pacientes na faixa etária de 18-29 anos (adultos jovens) tiveram significativamente mais solicitações de psicodiagnóstico, enquanto pacientes na faixa etária de mais 65 anos (idosos) tiveram significativamente menos solicitações. Nas outras categorias (escolaridade, estado civil e sexo), não houve diferença significativa entre os grupos $(p>0.05)$.

Tabela 1: Dados demográficos dos pacientes que realizaram psicodiagnóstico durante internação psiquiátrica em 2017.

\begin{tabular}{lc}
\hline \multicolumn{1}{c}{ Idade } & $\begin{array}{c}\text { N com } \\
\text { psicodiagnóstico }\end{array}$ \\
\hline $18-29$ anos & $17(41,5 \%)$ \\
$30-39$ anos & $9(22,0 \%)$ \\
$40-49$ anos & $9(22,0 \%)$ \\
$50-59$ anos & $3(7,30 \%)$ \\
$60-69$ anos & $3(7,30 \%)$ \\
\hline
\end{tabular}

Tabela 1: Dados

\begin{tabular}{lc}
\hline \multicolumn{1}{c}{ Idade } & $\begin{array}{c}\text { N com } \\
\text { psicodiagnóstico }\end{array}$ \\
\hline $70-89$ anos & 0 \\
Total & $41(100 \%)$ \\
Sexo & \\
F & $18(43,9 \%)$ \\
M & $23(56,1 \%)$ \\
Total & $41(100 \%)$ \\
Estado civil & \\
Solteiro & $30(73,2 \%)$ \\
Casado & $8(19,5 \%)$ \\
Divorciado & $2(4,9 \%)$ \\
Separado & $1(2,4 \%)$ \\
Viúvo & 0 \\
Total & $41(100 \%)$ \\
Grau de Escolaridade & \\
lgnorado & $2(4,9 \%)$ \\
Nenhum & 0 \\
Ensino fundamental incompleto & $17(41,5 \%)$ \\
Ensino fundamental completo & $4(9,8 \%)$ \\
Ensino médio incompleto & $3(7,3 \%)$ \\
Ensino médio completo & $9(22 \%)$ \\
Ensino superior incompleto & $5(12,2 \%)$ \\
Ensino superior completo & $1(2,4 \%)$ \\
Total & $41(100 \%)$ \\
&
\end{tabular}

\section{Dados da internação}

O tempo médio de permanência dos pacientes que tiveram solicitação de psicodiagnóstico foi 33,19 dias, sendo o mínimo 9 dias e o máximo 124 dias, com predomínio de permanência entre 2 a 6 semanas. Comparando o tempo de internação entre os grupos de quem realizou psicodiagnóstico e de quem não realizou, não houve diferença significativa (teste Mann-Whitney, $p=0.504$ ).

O motivo de internação é um campo descritivo no prontuário. Dos 44 pacientes que tiveram solicitação de exame, $12 \%$ (5 pacientes) contavam com apenas um motivo, $36 \%$ (16 pacientes) contavam com dois e $36 \%$ (16 pacientes) com três, enquanto 16\% (7 pacientes) não tinham este campo preenchido. Os motivos que mais apareceram foram: sintomas depressivos (17 vezes), sintomas psicóticos (13 vezes), ideação suicida (12 vezes) e heteroagressão (12 vezes).

Considerando o sistema de Classificação Internacional de Doenças-10 (CID-10) ${ }^{11}$, o grupo de diagnóstico mais prevalente no momento da internação foi F30-F39 (Transtornos do Humor), com 27 indivíduos, seguido por F20-F29 (Esquizofrenia), com 14 indivíduos. Entretanto, 41\% (18 casos) 
houve alteração no diagnóstico entre o momento da internação e da alta hospitalar. Das 14 pessoas que internaram com diagnóstico de Esquizofrenia, mais da metade (65\% ou 9 pessoas) obteve outro diagnóstico na alta, como de Retardo Mental (33\% ou 3 pacientes) e de Transtornos do Humor (22\% ou 2 pessoas). Já considerando indivíduos que receberam diagnóstico na linha dos Transtornos do Humor no momento da internação (27 indivíduos), $74 \%$ (20 pacientes) mantiveram este diagnóstico na alta, $7 \%$ tiveram alta com diagnóstico no grupo da Esquizofrenia e 19\% tiveram outro diagnóstico na alta, como Transtornos de Personalidade e Transtornos Mentais Orgânicos.

\section{Dados do psicodiagnóstico}

Assim como os motivos de internação psiquiátrica, o campo demanda para psicodiagnóstico é descritivo. Das 41 solicitações de psicodiagnóstico liberados no sistema, 41,5\% (17 solicitações) contavam com apenas um motivo e $58,5 \%$ (24 solicitações) contavam com dois, totalizando 65 motivos. As principais demandas de psicodiagnóstico foram auxílio na investigação do funcionamento emocional e cognitivo do paciente ( 25 solicitações) e auxílio no planejamento terapêutico (22 solicitações). Outras demandas que apareceram foram avaliação da capacidade intelectual e diagnóstico diferencial de personalidade.

O tempo entre a internação do paciente e a solicitação do psicodiagnóstico pela equipe médica foi disperso, sendo o mínimo de menos de um dia e o máximo de 73 dias. A média $(M)$ foi de 12,40 dias com desvio padrão (DP) de 14,03 dias. Já o tempo entre a solicitação e a finalização do procedimento, com anexação do laudo no prontuário eletrônico, foi de $M=15,07$ dias e $D P=12,76$ dias, sendo o intervalo (I) de 2 a 82 dias. O número de consultas para realização deste exame foi $M=5,51, \mathrm{DP}=1,28$ e I $=2-8$ consultas. Já o número de pessoas entrevistadas foi $\mathrm{M}=2,20, \mathrm{DP}=1 \mathrm{el}=1-6$ pessoas.

Os instrumentos utilizados para avaliar cognição foram as Escalas Weschler (WAIS-III ou WASI), em 37 indivíduos (90\%), e R1 - Forma B, em 4 indivíduos (10\%). Considerando os 37 indivíduos que realizaram teste das Escalas Weschler, a capacidade cognitiva global (QIT) foi $\mathrm{M}=76,70 \mathrm{com} D P=16,10 \mathrm{e} \mathrm{I}=50$ 129 , o que indica dispersão com predomínio de QIs rebaixados. Quatorze indivíduos (37,8\%) apresentaram diferença significativa entre QI verbal e de execução, sendo que $78,5 \%$ destes (11 indivíduos) tiveram QI de execução significativamente inferior ao verbal. Os instrumentos utilizados para avaliar personalidade foram House-Tree-Person, Pirâmides Coloridas de Pfister, Rorschach e/ou Teste de Apercepção Temática, de acordo com demanda da avaliação e características individuais.

\section{DISCUSSÃO}

Dentre as características demográficas dos pacientes que realizaram psicodiagnóstico, ressalta-se a baixa escolaridade: $41,5 \%$ evadiram da escola no Ensino Fundamental. A literatura indica uma associação inversa entre nível educacional e ocorrência de doença mental ${ }^{12,13}$. As dificuldades cognitivas tanto podem ser um pródromo do desenvolvimento de transtornos mentais como uma consequência destes. Em levantamento realizado pela Organização para Cooperação e Desenvolvimento Econômico ${ }^{14}$, no ano de 2015 , constatou-se que a média de abandono escolar de jovens com transtorno mental é 12 pontos percentuais acima daqueles sem transtornos mentais, chegando a $25 \%{ }^{15}$.

Identificou-se maior concentração de solicitações de psicodiagnóstico para pacientes na faixa etária de 18-29 anos. É nesta época, muitas vezes, que aparecem os primeiros sintomas psiquiátricos e que ocorre a primeira internação psiquiátrica ${ }^{16}$. Ou seja, na primeira internação, quando do começo dos sintomas, a solicitação deste exame é estatisticamente mais significativa.

O tempo médio de internação neste estudo foi de 33,19 dias, com maior concentração entre 2 e 6 semanas. Este tempo mostrou-se mais prolongado em comparação ao referido pela literatura. Em estudo realizado no RS foi constatada uma diminuição do tempo de internação para pacientes com transtorno mental, passando de 31,4 dias no ano 2000, para 20,0 dias em 2010 ${ }^{17}$. De acordo com o Ministério da Saúde, o prazo médio de internação hospitalar na Psiquiatria para adultos é de 30 dias $^{18}$. Para compreender estas diferenças é preciso considerar as características da instituição ${ }^{19}$. O tempo de permanência pode ser reflexo da realidade assistencial da unidade de internação psiquiátrica em hospital geral. Além das condições psiquiátricas complexas, os sintomas clínicos também compõe o tratamento, havendo necessidade de acionar outras especialidades por consultoria. Por se tratarem de pacientes que muitas vezes tem dificuldade de acesso à rede básica de saúde, é durante a internação que conseguem obter essa assistência. Outro fator possivelmente associado ao tempo de internação refere-se à realização de alguns procedimentos específicos, como terapia de eletroconvulsoterapia (ECT) e estimulação magnética transcraniana, oferecidos em poucos locais da rede.

Identificaram-se nos prontuários distintos motivos de internação, sendo os mais frequentes sintomas depressivos, psicóticos, ideação/tentativa de suicídio e risco ou ato de heteroagressão. Este dado está de acordo com as indicações para internação psiquiátrica, a saber alteração significativa nas capacidades de juízo crítico e de contenção de impulsos, associado a condutas de risco a si e a outros ${ }^{20}$. Ou seja, 
Pereira et al.

a indicação de internação psiquiátrica não se baseia em diagnóstico ou sintomas isolados, mas na relação destes com o contexto e os riscos a que o indivíduo e a família estão expostos.

Os dois grupos de diagnósticos mais prevalentes foram Transtornos do Humor e Esquizofrenia. Estudos ${ }^{21,22}$ corroboram estes dados, sendo estas condições psiquiátricas as que demandam maior necessidade de atenção em fase aguda. Destaca-se que uma parcela significativa dos pacientes internados teve modificação no diagnóstico entre o momento da internação e da alta. Este dado reforça um dos objetivos de uma internação psiquiátrica: estabelecer um diagnóstico preciso que possibilite o acesso a tratamento adequado. $\mathrm{Na}$ área de saúde mental, estabelecer um diagnóstico com precisão é uma tarefa complexa devido à variação de manifestações de comportamentos que pode ocorrer em cada crise ou em cada indivíduo ${ }^{23}$.

Quase $18 \%$ da amostra teve solicitação de psicodiagnóstico. Este dado poderia aparentar uma contradição: se o estabelecimento do diagnóstico é importante em uma internação e o psicodiagnóstico é uma ferramenta importante para tal, por que este recurso é utilizado apenas para uma pequena parcela?

O contexto e as características da internação psiquiátrica, assim como do psicodiagnóstico, auxiliam no entendimento desta questão. As diretrizes para internações breves, conforme Portaria n.14824, assim como as prerrogativas da estratégia Choosing Wisely ${ }^{9}$ ressaltam a importância de que os exames solicitados se restrinjam aos necessários para aquele momento. Durante a internação psiquiátrica, os pacientes estão em momento vulnerável, marcado por exacerbação dos sintomas, fase aguda da doença e/ ou em processo de ajuste medicamentoso, aspectos que tendem a impactar o rendimento em testes padronizados. Esta realidade deve ser considerada ao cogitar a possibilidade de um psicodiagnóstico, visto que o contexto e as condições situacionais do indivíduo naquele momento tendem a influenciar os resultados ${ }^{20,25}$.

Além disso, internações com objetivos específicos, como ajuste medicamentoso ou determinadas terapêuticas, como ECT, dispensam este exame já que o propósito é o tratamento de quadros refratários. Por fim, considerando o número de reinternações, é comum pacientes terem realizado este procedimento em internações prévias. Estudo ${ }^{26}$ realizado neste mesmo hospital apontou para taxa de readmissões em $29,17 \%$. Somado a isso, quanto mais internações psiquiátricas, maior a probabilidade reinternação ${ }^{27}$.

Assim, a parcela que se beneficia de ter psicodiagnóstico realizado durante internação psiquiátrica é aquela que interna com objetivo de investigação diagnóstica e que precisa de um exame detalhado e dispendioso para estabelecimento de diagnóstico.
O psicodiagnóstico, portanto, auxilia no estabelecimento diagnóstico e no planejamento terapêutico ${ }^{6}$.

A dispersão do tempo entre internação e solicitação do psicodiagnóstico pode ser compreendida pela diversidade da internação psiquiátrica. Em alguns casos, a equipe assistente já conhece o paciente e logo percebe a necessidade deste exame. Na maioria das vezes, entretanto, é preciso um tempo para que a equipe conheça o paciente e possa avaliar a necessidade do psicodiagnóstico. Além disso, é necessário que o paciente esteja minimamente estável dos sintomas para que consiga responder aos questionamentos e realizar os testes. Por outro lado, os resultados são importantes para o delineamento do tratamento daí a necessidade de ser solicitado o quanto antes.

O tempo entre solicitação do psicodiagnóstico e anexação do laudo no prontuário eletrônico do paciente também foi disperso, o que está relacionado ao contexto em uma internação psiquiátrica. O psicodiagnóstico deve respeitar a rotina estruturada da unidade, com horários estabelecidos para refeições, atividades de grupo, reuniões clínicas, visita dos familiares, realização de procedimentos médicos, entre outros. Interrupções são frequentes tanto por intercorrências clínicas, como por outros exames. Além disso, apesar do paciente estar internado, há que se respeitar o tempo de concentração e produtividade, evitando estressá-lo ainda mais. Por fim, por se tratar de um hospital escola, os atendimentos muitas vezes são realizados por alunos e, então, supervisionados, o que também acarreta tempo ao processo.

A média do número de consultas para realização do psicodiagnóstico foi de 5,51 consultas. Em contexto clínico ou ambulatorial, são indicados de 6 a 12 encontros para realização do psicodiagnóstico ${ }^{28}$. Pode-se compreender o menor número de consultas no contexto de internação ao considerarmos tanto o tempo de consulta como o acesso informal ao paciente. Enquanto no ambulatório, as consultas tem em torno de 50 minutos, na internação este tempo é variável, dependendo do rendimento do paciente. Por vezes, uma consulta pode ter duração de até duas horas, se o paciente se mostra concentrado, disposto e rendendo na atividade. Além disso, em ambulatório, na maioria das vezes, o contato com o paciente é limitado às consultas. Já na internação, há outras possibilidades, como a recreação terapêutica, refeições e grupos terapêuticos, espaços valiosos para observação do funcionamento do indivíduo.

A média de pessoas entrevistadas foi de 2,2 pessoas, sendo que em 10 casos $(24,4 \%)$, apenas o próprio paciente foi entrevistado. Este dado ilustra a dificuldade de contato com as famílias para realização de entrevistas. Apesar da dificuldade de entrevistar familiares, o psicólogo tem acesso a olhares de outros membros da equipe sobre o paciente. Em reuniões com equipe assistencial, por exemplo, se obtém 
informações acerca do processo de autonomia ou de outras características do paciente. Essas fontes de informação, apesar de não computadas no número de pessoas entrevistadas para a realização do psicodiagnóstico, fornecem dados valiosos.

$O$ rendimento cognitivo global variou entre Extremamente Baixo e Superior, apresentando dispersão com predomínio de QIs rebaixados. Este fato aponta tanto para possível prejuízo cognitivo basal, assim como para a possibilidade de deterioro cognitivo, ou ainda alterações situacionais devido à crise aguda. O contexto e as condições situacionais impactam no rendimento de testes padronizados ${ }^{29}$, devendo ser considerados na interpretação dos mesmos.

Enfim, a internação psiquiátrica é um recurso da rede de alta complexidade, necessário para o momento de maior vulnerabilidade do paciente, quando está em risco e há esgotamento dos recursos extra hospitalares. Muitas vezes, é durante este período que o paciente terá o estabelecimento de diagnóstico específico e, consequentemente, tratamento adequado. Entretanto, o psicodiagnóstico foi solicitado para apenas $17,8 \%$ dos pacientes internados. Essa realidade pode ser explicada ao atentarmos para diretrizes institucionais e nacionais, que enfatizam uso racional de recursos e internações breves, assim como para características deste exame e do contexto de internação psiquiátrica.

A prática do psicodiagnóstico auxilia no entendimento do funcionamento global do paciente. Contudo, é um recurso técnico que precisa ser usado de forma estratégica no momento de crise aguda, devido a fatores que envolvem desde o funcionamento da própria unidade até o estado mental e emocional do indivíduo. Esta configuração se reflete na maior demanda de psicodiagnóstico para pacientes adultos jovens, possivelmente em primeira internação psiquiátrica e em processo de avaliação diagnóstica.

Entre as limitações deste estudo, destaca-se que foi realizado em apenas um centro, sendo este um hospital geral universitário do sul do Brasil. Ampliar esta visão para outros hospitais permitirá um panorama mais abrangente da realidade do Brasil com relação a este tipo de exame neste contexto, gerando dados para políticas públicas e critérios específicos para solicitação.

\section{Conflitos de Interesse}

The authors declare no conflicts of interest.

\section{REFERÊNCIAS}

1. Brasil. Lei $n^{\circ} 10.216$, de 6 de abril de 2001. Diário Oficial da União. 2001 Abr 9;1:2

2. Cardoso L, Galera SAF. Internação psiquiátrica e a manutenção do tratamento extra-hospitalar. Rev Esc Enferm USP. 2011;45(1):87-94.

3. Brasil, Ministério da Saúde, Secretaria Executiva. Legislação em saúde mental: 1990-2002. 3. ed. Brasília, DF; 2002

4. Lopes SRA, Amorim SF. Avaliação psicológica no hospital geral. In: Bruscato WL, Benedetti C, Lopes SRA, editores. A prática da Psicologia Hospitalar na Santa Casa de São Paulo: novas páginas em uma antiga história. São Paulo: Casa do Psicólogo; 2010. p. 59-67.

5. Fongaro ML, Sebastiani RW. Roteiro de avaliação psicológica aplicada ao hospital geral. In: Angerami-Camon VA, editor. E a psicologia entrou no hospital. São Paulo: Cengage Learning; 2013. p. 5-68.

6. Krug JS, Trentini CM, Bandeira DR. Conceituação do psicodiagnóstico na atualidade. In: Hutz CS, Bandeira DR
Trentini CM, Krug JS, editores. Psicodiagnóstico. Porto Alegre: Artmed; 2016. p. 18.

7. Batista MN, Borges L. Processo de avaliação no contexto da saúde. In: Sociedade Brasileira de Psicologia, Miyazaki MCOS, Teodoro MLM, Gorayeb R, editores. PROPSICO: Programa de Atualização em Psicologia Clínica e da Saúde: Ciclo 1. Porto Alegre: Panamericana; 2017. p. 141-68.

8. Conselho Federal de Psicologia (BR). Resolução $n^{\circ} 9$, de 25 de abril de 2018. Diário Oficial da União. 2018 Maio 2;1:170.

9. American Board of Internal Medicine. Choosing Wisely [Internet]. Philadelphia; 2018 [citado 2018 Nov 15]. Disponível em: http://www. choosingwisely.org/.

10. Laguardia, J, Martins MS, Castro IRS, Barcellos GB. Qualidade do cuidado em saúde e a iniciativa "Choosing Wisely". Rev Eletron Comun Inf Inov Saude. 2016;10(1):1-8.

11. Organização Mundial da Saúde. Classificação Estatística Internacional de Doenças e Problemas
Relacionados à Saúde Décima Revisão. 8. ed. São Paulo: Edusp; 2008.

12. Organização Mundial da Saúde. O relatório mundial de saúde 2001 saúde mental: nova compreensão, nova esperança. Genebra; 2001.

13. Patel V, Kleinman A. Poverty and common mental disorders in developing countries. Bull World Health Organ. 2003;81(8):609-15.

14. Organização para Cooperação e Desenvolvimento Econômico. Education at a Glance 2015: Brasil [Internet]. Paris; 2015 [citado 2018 Nov 15]. Disponível em: https://www. oecd.org/brazil/Education-at-a-glance2015-Brazil-in-Portuguese.pdf

15. Hoffmann MS, Leibenluft E, Stringaris A, Laporte PP, Pan PM, Gadelha A, Manfro GG, et al. Positive atributes buffer the negative associations between low intelligence and high psychopatholohy with educational outcomes. J Am Acad Child Adolesc Psychiatry. 2015;55(1):47-53.

16. Sadock BJ, Sadock VA, Ruiz P. Compêndio de Psiquiatria: Ciência do Comportamento e Psiquiatria Clínica. Porto Alegre: Artmed; 2016. 
17. Horta RL, Costa JSD, Balbinot AD, Watte G, Teixeira VA, Poletto S. Hospitalizações psiquiátricas no Rio Grande do Sul de 2000 a 2011. Rev Bras Epidemiol. 2015;18(4):918-29.

18. Brasil, Ministério da Saúde. Orientações para funcionamento e supervisão dos serviços de saúde mental. Brasília, DF: Ministério da Saúde; 1990.

19. Souza JC, Souza N, Magna LA. Tempo médio de hospitalização e categorias diagnósticas em hospital psiquiátrico. J Bras Psiquiatr. 2008;57(2):112-16.

20. Pacheco MA, Cataldo Neto $A$, Menezes F, Krieger CA, Bersano L, Gil A. Aspectos do funcionamento de uma unidade de internação psiquiátrica de um hospital geral. Rev Psiquiatr Rio Gd Sul. 2003;25(1):106-14.
21. Miranda CA, Tarasconi CV, Scortegagna SA. Estudo epidêmico dos transtornos mentais. Aval Psicol. 2008;7(2):249-57.

22. Picci RL, Arcidiacono MC, Carezana C, Giaretto RM, Paschetta E, Giglio $D$, et al. O serviço psiquiátrico de diagnóstico e tratamento no hospital geral: usuários e novas urgências. J Nurs Health. 2011;1(2):300-10.

23. Brasil, Ministério da Saúde. Reforma psiquiátrica e política de saúde mental no Brasil: Conferência Regional de Reforma dos Serviços de Saúde Mental: 15 anos depois de Caracas. OPAS. Brasília, DF; 2005.

24. Brasil, Ministério da Saúde. Portaria $n^{\circ}$ 148, de 31 de janeiro de 2012. Diário Oficial da União. 2012 Feb 1;1:33.

25. Xavier FMF, Chachamovich E. A influência do uso de fármacos no psicodiagnóstico. In: Hutz CS,
Bandeira DR, Trentini CM, Krug JS, editores. Psicodiagnóstico. Porto Alegre: Artmed; 2016. p. 126.

26. Baeza FLC, Rocha NS, Fleck MPA. Readmission in psychiatry within a year of discharge: The role of symptoms at discharge and postdischarge care in a Brazilian sample. Gen Hosp Psychiatry. 2018;51:63-70.

27. Silveira LHC, Rocha CMF, Rocha KB, Zanardo GLP. O outro lado da porta giratória: apoio comunitário e saúde mental. Psicol Estud. 2016;21(2):325-35.

28. Rigoni MS, Sá SD. O processo psicodiagnóstico. In: Hutz CS, Bandeira DR, Trentini CM, Krug JS, editores. Psicodiagnóstico. Porto Alegre: Artmed; 2016. p. 29-30.

29. Urbina S. Depoimento. In: Estanislau G, Bressan RA, editores. Saúde Mental na escola: o que os educadores devem saber. Porto Alegre: Artmed; 2014. p. 13.

Recebido: 2 fev, 2020 Aceito: 24 fev, 2021 